\title{
Adaptation of the CRISPR/Cas9 system for targeted manipulations of the human mitochondrial genome
}

\author{
Zakirova E.G. ${ }^{1 *}$, Vereshchagina N.A. ${ }^{2}$, Mazunin I.O. ${ }^{2}$, Orishchenko K.E. ${ }^{1,2}$ \\ ${ }^{1}$ Institute of Cytology and Genetics, SB RAS, Novosibirsk, Russia \\ ${ }^{2}$ I. Kant Baltic Federal University, Kaliningrad, Russia \\ *e-mail: zakirova@bionet.nsc.ru
}

Key words: mitochondrial DNA editing, cybrids, MitoCRISPR/Cas9

Motivation and Aim: Repopulation of healthy copies of mtDNA in a multi-copy mitochondrial genome can be done using various technologies based on site-specific nucleases. Mitochondria-imported restriction endonucleases, ZFN and TALE-nucleases have already demonstrated the possibility of eliminating mutant copies of mtDNA, but the well-known CRISPR/Cas9 system have not been adapted for this task yet. Here we propose a strategy for modifying the components of the CRISPR/Cas9 to manipulate mitochondrial DNA (mtDNA) haplotype level in a cell.

Methods and Algorithms: A stable and uniform distribution of Cas9 nuclease among cells was obtained by integrating the Cas9 gene, that contained the mitochondrial localization signal on 5'-end, into the genome of cybrid cell lines NARP3-1 and NARP3-2. To transfer a guide RNA (gRNA) - the second component of the CRISPR / Cas9 system we added mitochondria-specific determinants of import of nucleic acids into the scaffold of gRNA. We checked, whether these modifications affected the functional activity of the system. Genetic constructs were designed in SnapGene software. The plasmids were assembled using the classical methods of molecular cloning and the Gibson assembly cloning method (NEB, USA). Integration of the nuclease gene was performed by Sleeping Beauty transposon system.

Results: We confirmed intramitochondrial localization of the modified nuclease, expressed from the cell nucleus, by immunocytochemistry analysis of the obtained stable cell lines NARP3-1-MitoCas9 and NARP3-2-MitoCas9. In vitro cleavage analysis of the template DNA using ribonucleoprotein complex showed that both of the guide RNA, without determinant in the RNA scaffold (NEG), and the modified variants of gRNA do not change the functional activity of the CRISPR/Cas9 system.

Conclusion: The derived transgenic cybrid cell lines allow performing further analysis of the effect of the MitoCRISPR / Cas9 system on the functional level without additional transfection with a plasmid encoding nuclease. Also we found that the CRISPR / Cas9 system remains functional activity after modifications in gRNA structure. Our results contribute to the further study of innovative technology of mitochondrial pathologies treatment - the MitoCRISPR/Cas9 system.

Acknowledgements: The work was supported by the Russian Foundation for Basic Research (19-015-00084) and the State budget project (0324-2019-0042). 